\title{
Private observatories seek public funding
}

[WASHINGTON] Privately run US observatories possessing high-performance telescopes but not the funds to equip them with state-of-the-art instruments have come up with a plan to persuade the National Science Foundation (NSF) to help pay for instrumentation.

The directors of the top observatories, which include the McDonald Observatory in Texas, the Steward Observatory in Arizona and the Harvard Smithsonian Center for Astrophysics, have suggested that the NSF should provide up to $\$ 8$ million a year in matching funds to pay for the instruments.

The plan has been agreed by a coordinating council of the directors of these observatories and of the National Optical Astronomy Observatories (NOAO). The NOAO is run by a consortium for the NSF and would be allowed to compete with the private facilities for matching instrumentation funds. The plan would replace the Facilities Instrumentation Program, under which the NSF pays for instruments and the observatories in exchange make telescope time available to outside researchers.

This programme was proposed three years ago by a panel of the National Research Council (NRC) chaired by Richard McCray of the University of Colorado. But it is virtually moribund because the observatories are reluctant to release the time in exchange for NSF support. The observatories' alternative - called the Revised Facilities Instrumentation Program - was due to be considered at a meeting of the NRC's Committee on Astronomy and Astrophysics in Washington this Tuesday (10 November).

The directors hope that the committee,

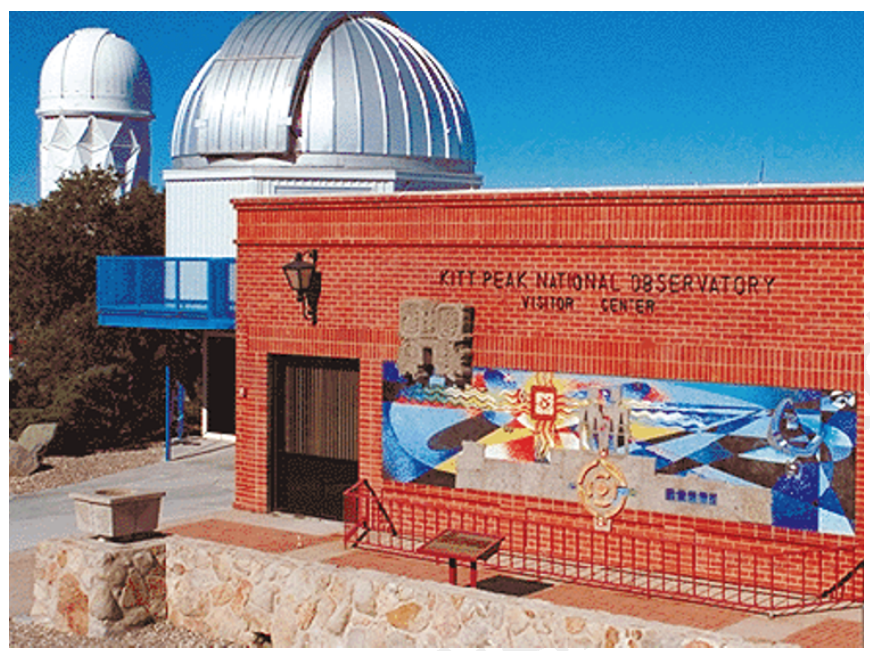

Still in the line of sight: The Kitt Peak

Observatory is currently the only facility in the United States with 4-metre-class telescopes that are open to all astronomers. which acts as a de facto advisory board for the NSF astronomy programme, will endorse their proposal in time for its inclusion in the NSF's next budget proposal, for the year 2000, to be finalized in February.

But the committee is more likely to study the proposal for a couple of months and, according to an official close to the proposal, then to back a compromise allowing the NSF to fund instruments on the basis of contributions of time or cash, or a mixture of the two.

Joseph Miller, director of the Lick Observatory in California and chairman of the committee of observatory directors that created the proposal, says the instrumentation is needed to keep US telescopes competitive with others, such as the European Southern Observatory in Chile. He denies that the plan could be construed as self-serving. "We have a \$200-million facility in Hawaii, which didn't cost the NSF a cent, but for $\$ 2$ million

\section{Changes in store for Japan's vet colleges}

[токуо] In a bid to improve the falling standard of veterinary education in Japan, the association representing veterinary schools has proposed a drastic reorganization of university veterinary colleges.

The association suggests that veterinary colleges at eight national universities be merged to create two schools at Kyushu University in southern Japan and Tohoku University in the north. These would each absorb four existing departments, leaving Tokyo and Hokkaido as the only other national universities with veterinary colleges. Plans released last month say the mergers should be completed by 2001 .

Hideaki Karaki, president of the association and professor of veterinary pharmacology at Tokyo University, says that "a shortage of staff makes it difficult to train good clinicians", although the existing courses provide good training in basic research.

The broadening of veterinary sciences, caused by the emergence of prion diseases and other cross-species transmission, has provoked concern over the poor standard of veterinary scientists in Japan. "There is a great demand for highly trained researchers and clinicians in areas such as public health and virology," says Karaki. "The proposed merger should create an integrated system to provide balanced education in preclinical and clinical veterinary medicine."

But the closures of veterinary colleges are likely to affect local agricultural industries, and may face strong local opposition. The government says that applications to veterinary colleges at national universities have trebled in the past decade - and that the prospect of even greater competition is likely to make the proposed changes unpopular.

Asako Saegusa they could vastly increase its [power]," he says, referring to the Keck telescopes.

But the revised proposal would clearly neglect a primary objective of the McCray panel, namely, to gain access to the best private telescopes for researchers from other institutions. At present they must depend on NOAO's ageing array of optical telescopes at Kitt Peak in Arizona. When the McCray panel reported in 1995, it was widely expected that NOAO would close several telescopes because of expected cuts in the NSF's overall budget. The budget has since grown, and most of the telescopes are still operating.

Still, supporters of the McCray proposal were expected to tell the NRC committee this week that the original idea of getting the observatories to free up time for outside researchers in exchange for NSF support remains sound. They admit it hasn't worked so far - only two small instruments have been supported by the NSF, and no more are pending. But they say that lukewarm support from the agency, combined with what appears to have been a tacit agreement among some observatory directors not to apply for the support on the available terms, have killed interest in it.

Several directors, for their part, remain adamantly opposed to the idea of swapping time for NSF support. They fear a 'slippery slope' by which the NSF would start to ask for telescope time in exchange for other grants. They also argue that releasing time to the federal agency would anger the founders who paid for the telescopes.

The disagreement reflects a history of bad blood between the 'haves' and the 'havenots' in US optical astronomy. "The NSF spends two-thirds of its astronomy budget on the national observatories - but many of us feel that the grants programme is really the heart of the discipline," says Miller. The grants programme supports many of the top astronomers and astrophysicists using the private facilities.
Colin Macilwain 